\title{
THE CLOSED IMAGE OF A METRIZABLE SPACE IS $M_{1}$
}

\author{
F. G. SLAUGHTER, JR.
}

\begin{abstract}
J. Ceder introduced the notions of $M_{1}$ space (a regular space with $\sigma$-closure preserving base) and stratifiable space as natural generalizations of Nagata and Smirnov's conditions for the metrizability of a regular space. Even though a topological space $Y$ which is the image of a metrizable space under a closed, continuous mapping need not be metrizable, we show as our main result that $Y$ will have a $\sigma$-closure preserving base. It follows that one cannot obtain an example of a stratifiable space which is not $M_{1}$ by constructing a quotient space from an upper semicontinuous decomposition of a metric space. In the course of establishing our major result, we obtain conditions under which the image of certain collections of sets under a closed, continuous mapping will be closure preserving.
\end{abstract}

1. Introduction. It is well known ([5] [6]) that the image of a metrizable space under a closed, continuous mapping need not be metrizable even though such a space will have considerable structure. Indeed, the closed, continuous images of metric spaces (now called Lašnev spaces) were characterized internally by Lašnev [4]. Although the Nagata-Smirnov metrization theorem makes it clear that a nonmetrizable Lašnev space will not have a $\sigma$-locally finite base; nevertheless, as we show in Theorem 6 , every Lašnev space has a $\sigma$-closure preserving base.

Regular spaces with $\sigma$-closure preserving bases were studied by $\mathrm{J}$. Ceder [2] who called them $M_{1}$ spaces. In the same paper, Ceder also defined the class of stratifiable spaces (which he called $M_{3}$ spaces) and showed that metrizable spaces are $M_{1}$ spaces and $M_{1}$ spaces are stratifiable. However, Ceder's question of whether a stratifiable space is also an $M_{1}$ space remains open. In searching for an example which is stratifiable but not $M_{1}$, it is natural to investigate the effect of applying a mapping $f$ which preserves stratifiability to a suitable $M_{1}$ space $X$ in the hope that $f(X)$ would fail to be $M_{1}$. Theorem 6 of course shows that one cannot obtain a stratifiable space (the closed, continuous image of a stratifiable space is

Presented to the Society, November 20, 1971; received by the editors May 2, 1972. AMS (MOS) subject classifications (1970). Primary 54C10, 54E20, 54E35.

Key words and phrases. $M_{1}$ space, $\sigma$-closure preserving base, closed mapping, Lašnev space.

(C) American Mathematical Society 1973 
stratifiable $\left[1\right.$, Theorem 3.1, p. 5]) which is not an $M_{1}$ space as the closed, continuous image of a metrizable space.

The major purpose of this paper is to obtain Theorem 6. In the course of proving Theorem 6, we obtain Lemma 3 and Lemma 4 which give conditions under which the closed image of certain collections of sets will be closure preserving.

2. Definitions and notation. Recall that a regular topological space $X$ is an $M_{1}$ space if there is a sequence $\left\{\mathscr{B}_{i}\right\}$ of open collections each of which is closure preserving and for which $\mathscr{B}=\bigcup\left\{\mathscr{B}_{i}: i=1,2, \cdots\right\}$ is a base for $X$.

Let $\mathscr{U}=\left\{U_{\alpha}: \alpha \in A\right\}$ be a collection of subsets of the set $X$. For any $B \in P(A), P(A)$ being the collection of all subsets of $A$, define $V(B)$ by $V(B)=\bigcup\left\{U_{\alpha}: \alpha \in B\right\}$. Suppose $f$ is a function from the set $X$ into the set $Y$. For $y \in Y$, we sometimes denote $f^{-1}(y)$ by $\tilde{y}$. Also if $T \subseteq X$, the saturated part of $T, T_{s}$, is defined by

$$
T_{s}=\bigcup\left\{f^{-1}(y): f^{-1}(y) \subseteq T\right\} .
$$

For the collection $\mathscr{U}=\left\{U_{\alpha}: \alpha \in A\right\}$ of subsets of $X$, we can consider the collection $\mathscr{W}(\mathscr{U})$ of all saturated parts of unions of sets in $\mathscr{U}$ i.e.

$$
\mathscr{W}(\mathscr{U})=\left\{W(B)=[V(B)]_{s}: B \in P(A)\right\} .
$$

Also set $\mathscr{Z}(\mathscr{U})=\{f(W): W \in \mathscr{W}(\mathscr{U})\}$. The notation $V(B), \mathscr{W}(\mathscr{U})$, $\mathscr{Z}(\mathscr{U}), \tilde{y}$ will be used throughout this paper.

If $Y$ is a topological space, $Y_{0} \subseteq Y$, and $\mathscr{B}$ a collection of open subsets of $Y$, we say that $\mathscr{B}$ is a base for the points of $Y_{0}$ provided that for any $y \in Y_{0}$ and open set $O$ containing $y$, there is a $B \in \mathscr{B}$ with $y \in B \subseteq O$.

All mappings in this paper are at least continuous.

\section{Preliminary results.}

LEMMA 1. Let $M$ be a metrizable space and let $f$ be a closed mapping from $X$ onto $Y$. Let $Y_{0}=\left\{y: f^{-1}(y)\right.$ is compact $\}$. Then there is a base $\mathscr{B}$ for the points of $Y_{0}$ which is $\sigma$-locally finite.

Proof. Let $\rho$ be a metric on $M$ compatible with the topology of $M$. Let $\left\{\mathscr{U}_{i}\right\}$ be a sequence of open covers of $M$ with mesh $\mathscr{U}_{i}$ going to 0 . For each $i$ let $\mathscr{U}_{i}=\left\{U_{i, \alpha}: \alpha \in A_{i}\right\}$ and for $j \geqq 1$, let

$$
\mathscr{V}_{i, j}=\left\{U_{i, \alpha_{1}} \cup U_{i, \alpha_{2}} \cup \cdots \cup U_{i, \alpha_{j}}: \alpha_{k} \in A_{i} \text { and all } \alpha_{k} \text { distinct }\right\} .
$$

Thus $\mathscr{V}_{i, j}$ consists of all exactly $j$-fold unions of elements of $\mathscr{U}_{i}$. Consider $\mathscr{C}_{i, j}=\left\{f\left(V_{s}\right): V \in \mathscr{V}_{i, j}\right\}$ where $V_{s}$ is the saturated part of $V$. Clearly since $f$ is a closed mapping, $\mathscr{C}_{i, j}$ is a collection of open subsets of $Y$. 
Claim. For any $y \in Y_{0}$ and $O(y)$ an open neighborhood of $y$, there are an $i$ and a $j$ so that $y \in S t\left(y, \mathscr{C}_{i, j}\right) \subseteq O(y)$.

To establish the claim, note that since $f^{-1}(y)$ is compact,

$$
\rho\left(f^{-1}(y), X-f^{-1}(O(y))\right)=\varepsilon>0 .
$$

Choose $i$ so that mesh $U_{i}<\varepsilon$. Also choose a minimal $j$ so that $f^{-1}(y)$ is covered by $j$ distinct sets of $\mathscr{U}_{i}$. Suppose that $U_{i, \alpha_{1}}, U_{i, \alpha_{2}}, \cdots, U_{i, \alpha_{i}}$ are any $j$ distinct sets of $\mathscr{U}_{i}$ which cover $f^{-1}(y)$. Then since $j$ is minimal, each $U_{i, \alpha}$ must hit $f^{-1}(y)$. Thus

$$
f^{-1}(y) \subseteq U_{i, \alpha_{1}} \cup U_{i, \alpha_{2}} \cup \cdots \cup U_{i, \alpha_{j}} \subseteq f^{-1}(O(y)) .
$$

It follows that there is a $C \in \mathscr{C}_{i, j}$ containing $y$. Moreover if $C^{\prime}$ is any element of $\mathscr{C}_{i, j}$ containing $y$, then $C^{\prime} \subseteq O(y)$. Hence $y \in \operatorname{St}\left(y, \mathscr{C}_{i, j}\right) \subseteq O(y)$.

For each $i, j$ let $R_{i, j}=\bigcup\left\{C: C \in \mathscr{C}_{i, j}\right\}$. Since $Y$ is a normal space whose open sets are $F_{\sigma}$ 's, for each $i, j$ we have countably many open sets $\left\{S_{i, j, k}: k=1,2, \cdots\right\}$ with

$$
\bigcup\left\{S_{i, j, k}: k=1,2, \cdots\right\}=\bigcup\left\{\mathrm{Cl}\left(S_{i, j, k}\right): k=1,2, \cdots\right\}=R_{i, j} .
$$

Using paracompactness of $Y$, each $\mathscr{C}_{i, j}$ restricted to $S_{i, j, k}$ has an open refinement $\mathscr{B}_{i, j, k}$ which covers $S_{i, j, k}$ and is locally finite in $Y$. Setting $\mathscr{B}=\bigcup\left\{\mathscr{B}_{i, j, k}: i, j, k=1,2, \cdots\right\}$, we have a $\sigma$-locally finite base for the points of $Y_{0}$ as desired.

Note that with the help of the Nagata-Smirnov metrization theorem and Lemma 1, we obtain the well-known results of Morita-Hanai [5, Theorem 1, p. 11] and Stone [6, Theorem 1, p. 691] that a perfect image of a metric space is metrizable.

Lemma 2 often appears implicitly in the literature concerning closed mappings; consequently its proof will be omitted.

LEMMA 2. Let f be a closed mapping from the $T_{1}$ space $X$ into the space $Y$. Let $\left\{y_{i}\right\}$ be a sequence of distinct points of $Y$ converging to $y$. Then any subsequence $\left\{x_{i_{j}}\right\}$ with $x_{i_{j}} \in \tilde{y}_{i_{j}}$ has a cluster point.

It will be useful in what follows to have conditions under which the closed image of a collection of sets will be closure preserving. Lemmas 3 and 4 provide such conditions.

LEMMA 3. Let $f$ be a closed mapping from the $T_{1}$ space $X$ into the Fréchet and Hausdorff space $Y$. Let $\mathscr{U}$ be an hereditarily closure preserving collection in $X$. Then $\mathscr{Z}(\mathscr{U})$ is closure preserving.

ProOF. Let $\mathscr{Z}^{\prime} \subseteq \mathscr{Z}(\mathscr{U})$. For suitable $\mathscr{Q} \subseteq P(A)$ we have $\mathscr{Z}^{\prime}=$ $\left\{f\left([V(B)]_{s}\right): B \in \mathscr{Q}\right\}$. Set $S=\bigcup\left\{Z: Z \in \mathscr{Z}^{\prime}\right\}$ and let $y \in \mathrm{Cl}(S)-S$. Since $Y$ is a Hausdorff and Fréchet space, there is a sequence $\left\{y_{i}\right\}$ of distinct 
points of $S$ converging to $y$. Each $\tilde{y}_{i}$ is contained in a $V\left(B_{i}\right)$ for suitable $B_{i} \in \mathscr{Q}$. For each $i=1,2, \cdots$, define $C_{i}$ by

$$
C_{i}=\left\{\alpha: \alpha \in B_{i} \text { and } U_{\alpha} \cap \tilde{y}_{i} \neq \varnothing\right\} .
$$

Clearly $\tilde{y}_{i} \subseteq V\left(C_{i}\right)$. Moreover we will show that at most finitely many $C_{i}$ 's are infinite. Clearly if this is not the case, then there is a sequence of distinct $\alpha_{i_{j}}$ 's with $\alpha_{i_{j}} \in C_{i_{j}}$. Choosing points $x_{i_{j}} \in U_{\alpha_{j}} \cap \tilde{y}_{i_{j}}$, we have a discrete sequence in violation of Lemma 2.

Without loss of generality, assume that each $C_{i}$ is finite. Indeed we have that $C=\bigcup\left\{C_{i}: i=1,2, \cdots\right\}$ is finite; if not again choose a discrete sequence of points from $\tilde{y}_{i_{j}} \cap U_{\alpha_{i_{j}}}$ for suitable distinct $\alpha_{i_{j}}$ 's and $y_{i_{j}}$ 's in violation of Lemma 2.

Thus the range of the mapping $\tilde{y}_{i} \rightarrow C_{i}$ lies in the finite set $P(C)$. Consequently there is $C_{i_{0}}$ and sequence $C_{i_{j}}$ with $C_{i_{0}}=C_{i_{j}}$ for $j=1,2, \cdots$. Then for each $j, \tilde{y}_{i_{j}} \subseteq V\left(C_{i_{j}}\right)=V\left(C_{i_{0}}\right) \subseteq V\left(B_{i_{0}}\right)$. Thus $y \in \mathrm{Cl}\left(f\left(V\left(B_{i_{0}}\right)_{s}\right)\right)$ from which it follows that $\mathscr{Z}^{\prime}$ is closure preserving.

We note that $D$. Lutzer has generalized Lemma 3 by deleting the assumption that $X$ is $T_{1}$ and $Y$ is Hausdorff.

By requiring the mapping $f$ in Lemma 3 to be perfect and the collection $\mathscr{U}$ to be locally finite, we can obtain the conclusion of Lemma 3 without restriction on $Y$. More precisely, we have

LEMMA 4. Let $f: X \rightarrow Y$ be a perfect mapping (i.e. a closed mapping with compact fibers) and let $\mathscr{U}$ be a locally finite collection of subsets of $X$. Then $\mathscr{Z}(\mathscr{U})$ is closure preserving.

LEMMA 5. Let $f: M \rightarrow Y$ be a closed mapping from the metrizable space $M$ onto the space $Y$. Then each point $y$ in $Y$ has a closure preserving local base $\mathscr{D}_{y}$ of open neighborhoods.

Proof. Let $y$ in $Y$ be fixed. By choosing a metric $\rho$ on $M$ compatible with the topology of $M$, we can obtain a sequence $\left\{\mathscr{U}_{j}\right\}$ of locally finite open collections with mesh $\mathscr{U}_{j}<1 / j$ so that every element of each $\mathscr{U}_{j}$ hits $\tilde{y}$. For each $j$ let $\dot{U}_{j}=\left\{U_{j, \alpha}: \alpha \in A_{j}\right\}, \mathscr{V}_{j}=\left\{V(B): B \in P\left(A_{j}\right)\right\}$ and

$$
\mathscr{W}=\left\{\bigcup_{j=1}^{\infty} V_{j}: V_{j} \in \mathscr{V}_{j} \text { and } \bigcup_{j=1}^{\infty} V_{j} \supseteq \tilde{y}\right\} \text {. }
$$

We assert that $\mathscr{D}_{y}=\left\{f\left(W_{s}\right): W \in \mathscr{W}\right\}$ is a closure preserving system of open neighborhoods of $y$ which forms a local base at $y$.

Clearly $\mathscr{D}_{y}$ is a system of open neighborhoods of $Y$. Moreover it is easy to see that since $f$ is a closed mapping, $\mathscr{D}_{y}$ is a local base at $y$. It remains to show that $\mathscr{D}_{y}$ is closure preserving. To do so, let $\mathscr{D}^{\prime} \subseteq \mathscr{D}_{y}$ and let

$$
S=\bigcup\left\{D: D \in \mathscr{D}^{\prime}\right\}=\bigcup\left\{f\left(W_{s}\right): W \in \mathscr{W}^{\prime}\right\}
$$


for a suitable $\mathscr{W}^{\prime} \subseteq \mathscr{W}$. Let $z \in \mathrm{Cl}(S)-S$. Since $Y$ is a Fréchet space, there is a sequence $\left\{y_{i}\right\}$ of distinct points of $S$ converging to $z$. Choose $W_{i} \in \mathscr{W}^{\prime}$ so that $\tilde{y}_{i} \subseteq W_{i}$. For each $i$, we have $W_{i}=\bigcup\left\{V_{i, j}: j=1,2, \cdots\right\}$ where $V_{i, j} \in \mathscr{V}_{j}$.

Claim. There is an $m$ so that for all but finitely many $i$ 's, if $j>m$, then $V_{i, j} \cap \tilde{y}_{i}=\varnothing$.

To establish the claim suppose the contrary. Then there is a sequence $\left\{\tilde{y}_{i_{k}}\right\}$ so that $\left\{\tilde{y}_{i_{k}}\right\}$ hits $\bigcup\left\{V_{i_{k}, j}: j>k\right\}$ for each $k=1,2, \cdots$. Choose sequences $\left\{p_{i_{k}}\right\}$ and $\left\{q_{i_{k}}\right\}$ with $p_{i_{k}} \in \tilde{y}_{i_{k}}, q_{i_{k}} \in \tilde{y}$ and $\rho\left(p_{i_{k}}, q_{i_{k}}\right)<1 / k$. According to Lemma $2,\left\{p_{i_{k}}\right\}$ has a cluster point $p$. Hence some subsequence of $\left\{p_{i_{k}}\right\}$ converges to $p$, the image of this subsequence converges to $z$ and consequently $f(p)=z$. But since $\rho\left(p_{i_{k}}, q_{i_{k}}\right) \rightarrow 0$, a subsequence of $\left\{q_{i_{k}}\right\}$ converges to $p$ and $f(p)=y$. But $y \neq z$ and therefore the claim is established.

Having established the claim, without loss of generality we can assume there is an $m$ so that, for any $i, \tilde{y}_{i} \subseteq \bigcup\left\{V_{i, j}: 1 \leqq j \leqq m\right\}=O_{i}$. Each $\tilde{y}_{i}$, by virtue of being contained in $O_{i}$, lies in a union of elements from the locally finite collection $\mathscr{U}_{1} \cup \mathscr{U}_{2} \cup \cdots \cup \mathscr{U}_{m}$. By Lemma $3,\left\{f\left(\left[O_{i}\right]_{s}\right): i=1,2, \cdots\right\}$ is closure preserving and thus for some $i_{0}, z \in \mathrm{Cl}\left(f\left(\left[O_{i_{0}}\right]_{s}\right)\right)$. But $f\left(\left[O_{i_{0}}\right]_{s}\right)$ is contained in some $D \in \mathscr{D}^{\prime}$ and $z \in \bigcup\left\{\mathrm{Cl}(D): D \in \mathscr{D}^{\prime}\right\}$. Therefore $\mathscr{D}_{v}$ is closure preserving as desired.

4. Main result. In [2, Theorem 7.6, p. 117], Ceder established that the quotient space obtained by identifying a closed set in a metric space to a point is an $M_{1}$ space. Theorem 6 provides a considerably more general result.

THEOREM 6. Let $f$ be a closed mapping from the metric space $M$ onto the space $Y$. Then $Y$ is an $M_{1}$ space.

Proof. By [3, Theorem 1, p. 1504], $Y=Y_{0} \cup \bigcup\left\{Y_{i}: i=1,2, \cdots\right\}$ with $f^{-1}(y)$ compact for $y \in Y_{0}$ and each $Y_{i}$ discrete as a collection of singletons. Applying Lemma 1 , we have a sequence $\left\{\mathscr{B}_{i}\right\}$ of locally finite open collections in $Y$ with $\mathscr{B}=\bigcup\left\{\mathscr{B}_{i}: i=1,2, \cdots\right\}$ a base for the points of $Y_{0}$. Using collectionwise normality of $Y$, for each $i$ swell the points $y$ of $Y_{i}$ to a discrete collection $\mathscr{E}_{i}=\left\{E_{i, y}: y \in Y_{i}\right\}$ of open sets with $y \in E_{i, y}$. By Lemma 5 , each point $y$ of $Y_{i}$ has a closure preserving open base of neighborhoods $\mathscr{D}_{y}$. We may assume without loss of generality that the elements of $\mathscr{D}_{y}$ are subsets of $E_{i, y}$. Setting $\mathscr{F}_{i}=\bigcup\left\{\mathscr{D}_{y}: y \in Y_{i}\right\}$ we obtain a closure preserving base for the points of $Y_{i}$. Consequently

$$
\bigcup\left\{\mathscr{B}_{i}: i=1,2, \cdots\right\} \cup \bigcup\left\{\mathscr{F}_{i}: i=1,2, \cdots\right\}
$$

is a $\sigma$-closure preserving base for $Y$ and Theorem 6 is proved. 
Recall that the closed images of metric spaces have been characterized by Lašnev [4]. For such spaces, called Lašnev spaces, the implications metric space $\rightarrow$ Lašnev space $\rightarrow M_{1}$ space hold. Moreover, as an immediate consequence of Theorem 6 we obtain in Corollary 7 a partial solution to Ceder's problem of whether every subspace of an $M_{1}$ space is an $M_{1}$ space.

COROLlaRY 7. Every subspace of a Lašnev space is $M_{1}$.

\section{REFERENCES}

1. C. J. R. Borges, On stratifiable spaces, Pacific J. Math. 17 (1966), 1-16. MR 32 \#6409.

2. J. G. Ceder, Some generalizations of metric spaces, Pacific J. Math. 11 (1961), 105-126.

3. N. S. Lašnev, On continuous decompositions and closed mappings of metric spaces, Dokl. Akad. Nauk SSSR 165 (1965), 756-758=Soviet Math. Dokl. 6 (1965), 1504-1506. MR 33 \#703.

4. - Closed images of metric spaces, Dokl. Akad. Nauk SSSR 170 (1966), 505-507=Soviet Math. Dokl. 7 (1966), 1219-1221. MR 34 \#3547.

5. K. Morita and S. Hanai, Closed mappings and metric spaces, Proc. Japan Acad. 32 (1956), 10-14. MR 19, 299.

6. A. H. Stone, Metrizability of decomposition spaces, Proc. Amer. Math. Soc. 7 (1956), 690-700. MR 19, 299.

Department of Mathematics, University of Pittsburgh, Pittsburgh, PennsylVANIA 15213 